\title{
INVESTIGATION OF CIRCULATING SERUM microRNA-328-3p AND microRNA-3135a EXPRESSION AS PROMISING NOVEL BIOMARKERS FOR AUTISM SPECTRUM DISORDER
}

\author{
Popov $\mathrm{NT}^{1}$, Minchev $\mathrm{DS}^{2}$, Naydenov $\mathrm{MM}^{3}$, Minkov $\mathrm{IN}^{4}$, Vachev $\mathrm{TI}^{2,4, *}$ \\ *Corresponding Author: Assistant Professor Tihomir I. Vachev, Ph.D., Department of Plant Phisyology \\ and Molecular Biology, University of Plovdiv "Paisii Hilendarski," 24 Tzar Assen Str., Plovdiv, Bulgaria. \\ E-mail: tiho9@abv.bg
}

\begin{abstract}
Circulating microRNAs (miRNAs) are emerging as promising diagnostic biomarkers for autism spectrum disorder (ASD), but their usefulness for detecting ASD remains unclear. Nowadays, development of promising biomarkers for ASD remains a challenge. Recently, dysregulation of the miRNAs expression in postmortem brain tissue, serum and peripheral blood, have been associated with ASD. Circulating miRNAs are known to be secreted by a number of different cells and can interpose delivery of information into receiver cells, thus affecting their functions. Based on this fact, it is supposed that serum miRNAs could be a novel class of biomarkers for prognosis or diagnosis of pathological disorders including ASD. In the current research, we investigated whether the expression patterns of circulating miRNAs showed dysregulation in subjects diagnosed with ASD. Expression levels of serum miR-328-3p and miR-3135a were analyzed by quantitative reverse transcription polymerase chain reaction (qRT-PCR) method of subjects diagnosed with ASD in comparison with healthy control subjects. Our data showed that miR-328-3p and miR-3135a were substantially down-regulated in ASD patients than in those of healthy control subjects. Moreover, target gene analysis of altered serum miRNAs displayed that these molecules
\end{abstract}

\footnotetext{
${ }^{1}$ State Psychiatry Hospital Pazardzhik, Pazardzik, Bulgaria

2 Department of Plant Physiology and Molecular Biology, University of Plovdiv "Paisii Hilendarski," Plovdiv, Bulgaria

${ }^{3}$ Department of Human Anatomy and Physiology, University of Plovdiv "Paisii Hilendarski," Plovdiv, Bulgaria

${ }^{4}$ Institute of Molecular Biology and Biotechnologies (IMBB), Plovdiv, Bulgaria
}

targeted 162 genes denoted as unique validated targets in the miRWalk database, 71 of which appear to participate in biological pathways involved in synaptic pathways and neurodegenerative condition such as Alzheimer, Huntington and Parkinson diseases. Finally, the results strongly suggested that dys-regulated serum miRNAs might be involved in molecular pathways associated with ASD and miR-328-3p and miR-3135a have the potential to be promising novel biomarkers for ASD.

Keywords: Autism spectrum disorder (ASD); MicroRNA (miRNA) expression profiling; Serum miRNAs; Stem-loop quantitative reverse transcription polymerase chain reaction (qRT-PCR).

\section{INTRODUCTION}

MicroRNAs (miRNAs) are small, single-stranded, non coding RNA molecules that regulate gene expression of their complementary messenger RNA targets $[1,2]$. Taking into account, the significance of these regulatory molecules in gene regulation, discovery of dysregulated miRNAs could benefit to clarify the molecular differences of miRNAs serum profiles between the autism spectrum disorder (ASD) subjects and typically developing children (TDC). The prevalence of ASD has increased considerably (from 2-5/10,000 to 1:68 children) during the last two decades $[3,4]$. Increased attention and changes made to the diagnostic criteria by the medical community have necessarily contributed to this tendency [5], as well as several environmental factors, acting particularly over early postnatal neurodevelopment and prenatal development [6]. In addition, advanced parental age at time of conception has also been shown to pose a risk of ASD [7]. Despite major advances in the understanding of ASD pathogenesis, inter individual heterogeneity and the levels 
of complexity has largely complicated the delivery of extensive scientific learning into efficient clinical practices. Currently, the golden standard to diagnose ASD is built on information from several sources: family caregivers, teachers, standardized behavioral scales and clinical observations. Also, pervasive development of the patients is only monitored by clinical scales with uncertain reliability, especially in early age. Specific and sensitive quantitative biomarkers, evaluated through experimental methods, electrophysiological techniques and brain imaging, would greatly support clinicians in providing more timely referrals to behavioral intervention programs' earlier diagnoses and evidence-based prognosis [8]. Quantitative polymerase chain reaction (qPCR) technologies offer a accurate tools to search for specific miRNAs profiles applicable as potential biomarkers for neurodevelopmental disorders. The choice of miR-328-3p and miR-3135a as potential biomarkers in ASD is based mainly on our preliminary data showing differential expression changes of these miRNAs from small RNA sequencing. In addition, there is no scientific data on the role of these miRNAs in ASD, although the importance of many miRNA molecules has been shown in the etiopathogenesis of several neurological disorders. The current study was performed in order to shed light on the probable participation of miRNAs in ASD. The main aim of the current research was to analyze differential expression of miR-328-3p and miR-3135a in serum of ASD subjects and their relevance as potential blood-based biomarkers.

\section{MATERIALS AND METHODS}

Patients. A total of 30 subjects (24 males and six females) with ASD aged 3 to 11 years, and 30 TDC sex- and age-matched to the ASD group were included in this study. All participants were randomly selected from the family practices in the Plovdiv region. Probands were evaluated by certified psychiatrists and the diagnosis of ASD was made by clinical examination, Gilliam autism rating scale
(GARS), childhood autism rating scale (CARS) and autism diagnostic interview (ADI-R), adhering to the diagnostic and statistical manual of mental disorders (DSM V) criteria. The GARS norm-referenced screening instrument was used for ASD symptom assessment. To help differentiate subjects with ASD from those with other developmental delays, we used CARS. The ADR-R is a structured interview performed with the parents of the patients. This is the golden standard for assessment of ASD patients. The DSM-V is the standard classification of mental disorders used by mental health professionals and physicians in the USA and most research teams worldwide.

The control group representing TDC was assigned with the aim to match by sex and age to the ASD group. Inspection of all children in the healthy group for absence of autistic features was done by clinical examination and CARS. Children with known infectious, oncological, metabolic or genetic conditions were excluded from the study. No children were receiving any drug therapy when they were recruited.

Statement of Ethics. The Institutional Review Board of the Ethics Committee of the Medical University of Plovdiv approved the methodology of the study and the written informed consent forms.

Blood Processing and RNA Extraction. Blood was drawn by venipuncture from a peripheral vein while the participants were fasting ( $>3$ hours without a meal). Separation into blood cells and serum was done by centrifuging at $1600 \mathrm{~g}$ for $10 \mathrm{~min}$. RNA was extracted from the $200 \mu \mathrm{L}$ serum aliquots, with the addition of $5 \mu \mathrm{L}(100 \mathrm{nM})$ spikein cel-miR-39 control, added for internal standardization. Extraction of serum RNA was done using the PAXgene blood miRNA kit (PreAnalytiX GmbH, Hombrechtikon, Switzerland), following the manual purification of total RNA, including miRNA protocol, recommended by the manufacturer.

Quantification of Serum MicroRNAs. The Maxima First Strand cDNA Synthesis Kit (Thermo Scientific,

Table 1. MicroRNA-specific quantitative reverse transcription polymerase chain reaction primer sets.

\begin{tabular}{|l|l|}
\hline MiRNAs & \multicolumn{1}{c|}{ Primer Sequences (5'>3') } \\
\hline $\begin{array}{l}\text { miR-197-5p SL } \\
\text { miR-197-5p F }\end{array}$ & $\begin{array}{l}\text { CTC AAC TGG TGT CGT GGA GTC GGC AAT TCA GTT GAG CCT CCC AC } \\
\text { ACA CTC CAG CTG GGC GGG TAG AGA GGG CAG T }\end{array}$ \\
\hline $\begin{array}{l}\text { miR-500a-5p SL } \\
\text { miR-500a-5p F }\end{array}$ & $\begin{array}{l}\text { CTC AAC TGG TGT CGT GGA GTC GGC AAT TCA GTT GAG TCT ACC CC } \\
\text { ACA CTC CAG CTG GGT AAT CCT TGC TAC CTG G }\end{array}$ \\
\hline $\begin{array}{l}\text { miR-424-5p SL } \\
\text { miR-424-5p F }\end{array}$ & $\begin{array}{l}\text { CTC AAC TGG TGT CGT GGA GTC GGC AAT TCA GTT GAG TTC AAA AC } \\
\text { ACA CTC CAG CTG GGC AGC AGC AAT TCA TGT }\end{array}$ \\
\hline Cel-miR-39 Spike-in & UCA CCG GG GUA AAU UA \\
\hline $\begin{array}{l}\text { Cel-miR-39 SL } \\
\text { Cel-mi-39 F }\end{array}$ & $\begin{array}{l}\text { CTC AAC TGG TGT CGT GGA GTC GGC AAT TCA GTT GAG CAA GCT GA } \\
\text { ACA CTC CAG CTG GGT CAC CGG GTG TAA ATC }\end{array}$ \\
\hline Universal R & GTC GGC AAT TCA GTT GAG \\
\hline
\end{tabular}

MiRNAs: microRNAs; SL: stem-loop. F: forward; Spike-in: exogenous RNA control; R: reverse. 
Waltham, MA, USA) were used for miRNA specific cDNA synthesis. MicroRNA-specific primer sequences used in this study are shown in Table 1. MicroRNA specific cDNA $(5 \mu \mathrm{L})$ were subjected to pre amplification with peqGOLD Taq DNA Polymerase (VWR, Radnor, PA, USA) prior to the reverse transcription-(RT-PCR) step in order to enhance sensitivity of the test. The qRT-PCR was carried out using the Maxima SYBR Green qPCR Master Mix (Thermo Scientific) and the ABI PRISM ${ }^{\circledR} 7500$ system (Applied Biosystems, Foster City, CA, USA). All the experiments were performed in duplicate. Each sample was normalized using spiked-in control and relative quantification of miRNAs were calculated applying the $2^{-\Delta \Delta \mathrm{Ct}}$ method. Statistical analyses were made by the Statistical Package for the Social Sciences (SPSS) software, version 20.0 (SPSS $^{\circledR}$; IBM Inc., Armonk, NY, USA). The analysis of variance (ANOVA) $t$-test on the data of $\mathrm{Ct}$ values was used for investigation of dysregulation of the analyzed miRNAs between TDC and ASD groups. MedCalc statistical (https://medcale.org/) software was used to perform receiver operating characteristic (ROC) analysis.

Pathways Prediction Analysis of Differentially Expressed miR-328-3p and miR-3135a Serum. First, we managed to obtain every validated target gene available in the miRWalk 2.0 database (zmf.umm.uni-heidelberg. $\mathrm{de} / \mathrm{apps} / \mathrm{zmf} / \mathrm{mirwalk2/)}$ for both of the serum miRNAs we studied. The miRWalk database offers a convenient direct search option, but only for those pathways in which putative target genes take part. However, such a direct search option is not available for validated target genes. We developed our own script that uses a list of validated target genes as a query and automatically explores the Kyoto Encyclopedia of Genes and Genomes (KEGG) database (http://www.genome.jp.kegg) for pathways in which our validated target genes participate. This made it possible to gather information for all of the validated targets without searching the whole KEGG database by hand. Afterwards, the number of target genes was plotted against their respective pathways on separate diagrams for each miRNA we investigated.

\section{RESULTS}

Identification of MicroRNA Deregulation Signature in Autism Spectrum Disorder. We analyzed the expression of two serum miRNAs using the stem-loop qRT-PCR assay. Amplicon-specific PCR amplification corresponding to miR-3135a and $\mathrm{miR}-328-3 \mathrm{p}$ was confirmed by melting curve analysis (Figure 1). Our data indicate changes in relative expression levels of miR-3135a and miR-328-3p, which were markedly lower in ASD patients than those in TDC, and the relative serum levels of these miRNAs could distinguish ASD from healthy control patients (Figure 2). To assess the ability of the analyzed serum miRNAs as promising biomarkers for ASD, we performed a ROC analysis, and the area under curve (AUC) was measured (Figure 3). Our relative qRT-PCR data suggests that miR-3135a and miR-328-3p are significantly down-

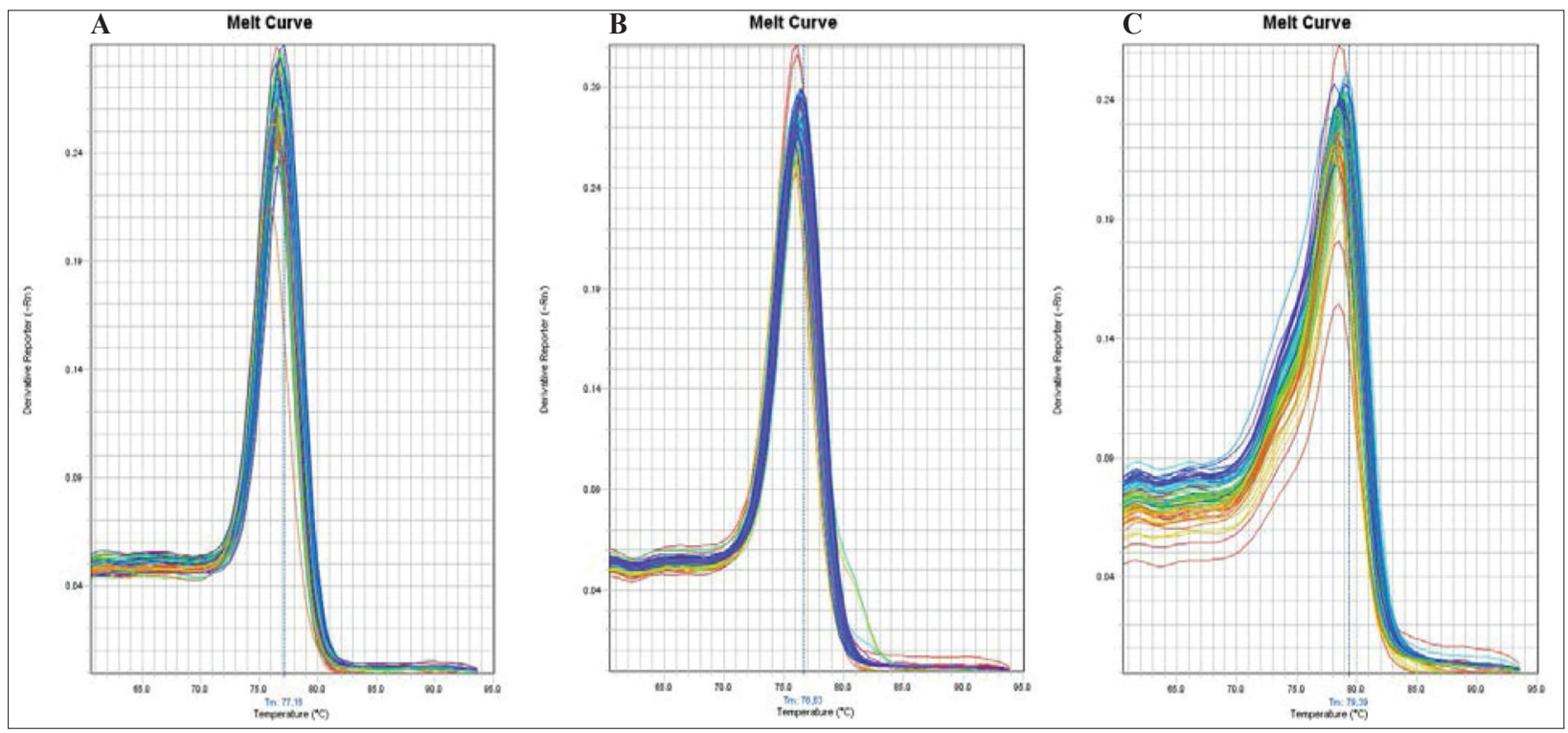

Figure 1. The qPCR data showing a DNA melt profile result for amplification of the specific single product in qRT-PCR analysis. Specific single products corresponding to exogeneous spike-in control cel-miR-39 (panel A); miR-328-3p (panel B) and miR-3135a in ASD and healthy control patients, were confirmed by monitoring the dissociation curve (melting curve analysis). The melting temperatures of miR-3135a amplicons were $76 \pm 1{ }^{\circ} \mathrm{C}$ (panel B), whereas spiked-in cel-miR-39 control had a melting temperature of $77 \pm 1{ }^{\circ} \mathrm{C}$ (panel A), and melting temperatures of miR-328-3p amplicons were $79 \pm 1{ }^{\circ} \mathrm{C}$ (panel C), respectively. 


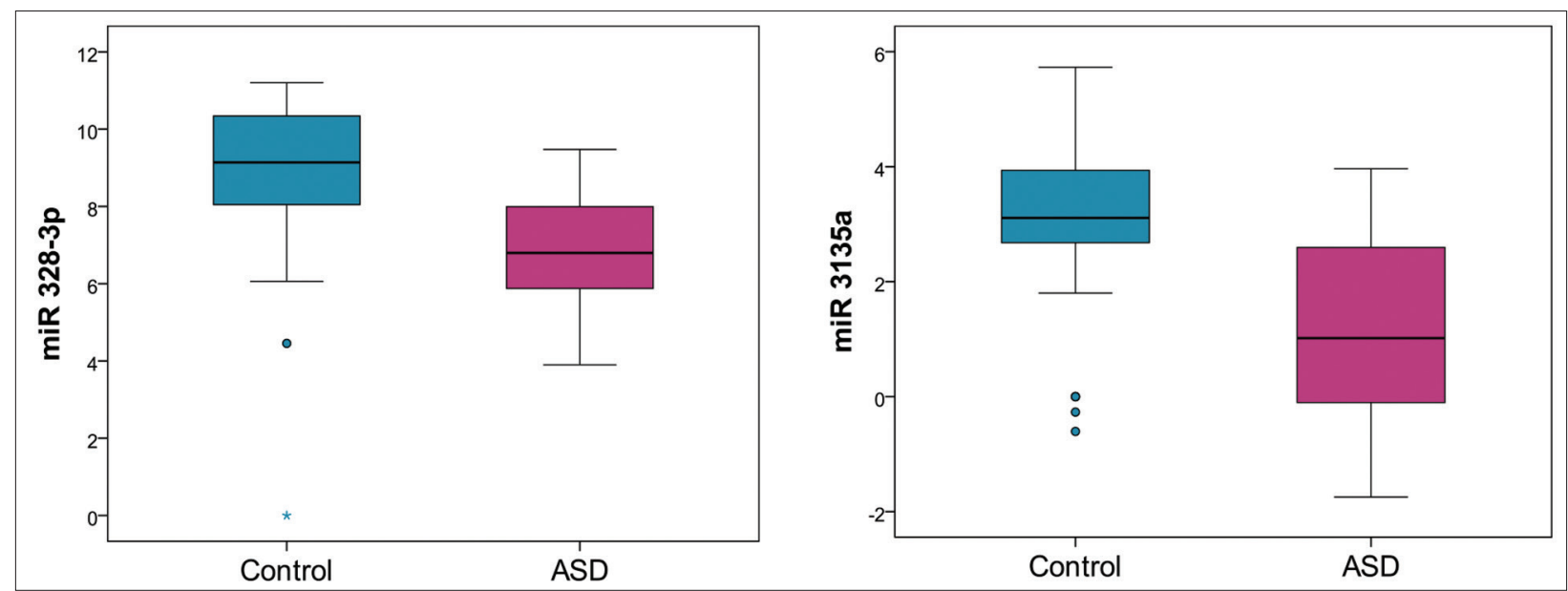

Figure 2. Differential expression of serum miRNAs in ASD patients. Quantitative RT-PCR analysis of miR-3135a and miR-328-3p levels. The circulating serum miRNAs signatures were identified by miRNA-specific stem-loop qRT-PCR analysis in the ASD and control groups. Expression levels of the analyzed miRNAs were normalized to spiked-in cel-miR-39 control and expressed in relation to controls.
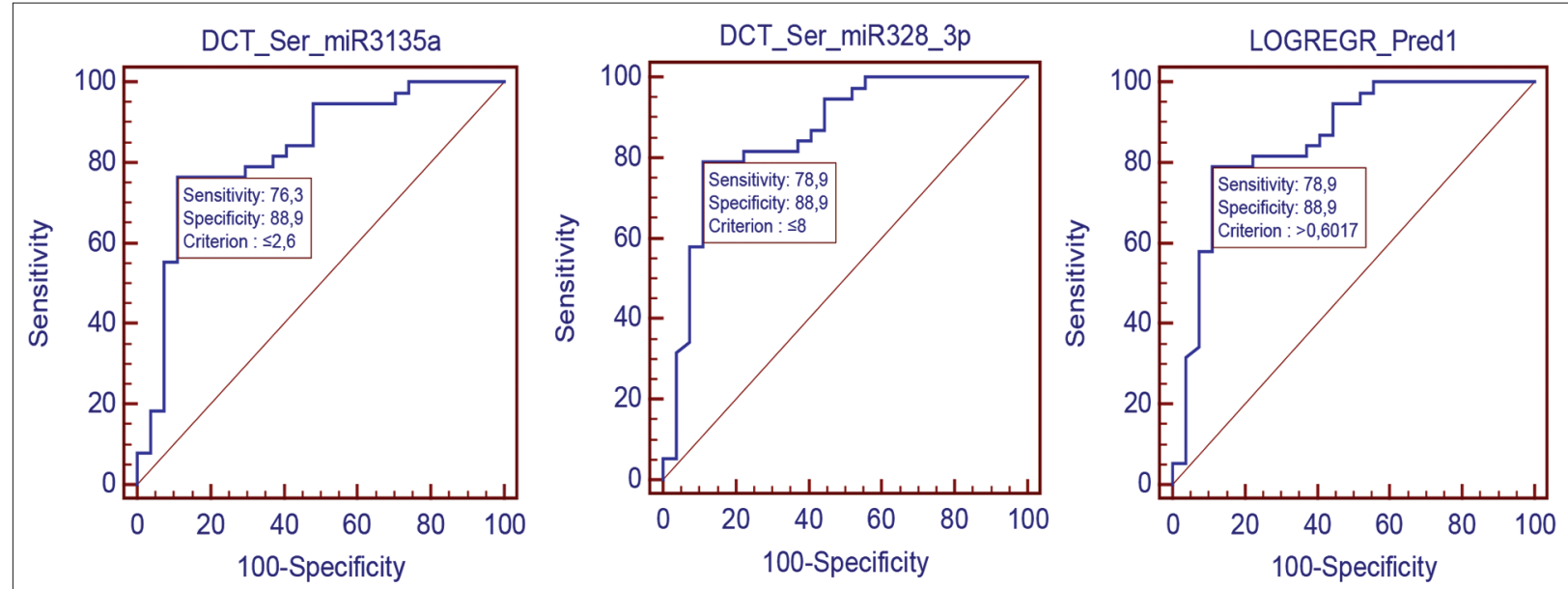

Figure 3. Receiver operating characteristic curve analysis using differentially expressed serum miRNAs. The ROC for miR-3135a, and miR-328-3p signature in patients with ASD was performed to evaluate the prediction accuracy of selected biomarkers. The dotted diagonal line represents random classification accuracy (AUC 0.5). The ROC curves were drawn for miR-3135a, and miR-328-3p, which yielded 0.828 and 0.858 as AUC values, respectively. Combined ROC curve describe the logistic regression (LOGREGR) of the differentially expressed miRNA members (miR-3135a and miR-328-3p). Diagnostic sensitivity of combined classifiers was $78.9 \%$ with the corresponding specificity of $88.9 \%$. The combination of the miRNAs showed a correspondence to that using only miR-328-3p as a biomarker.

regulated in ASD patients (Figure 4). The $t$-test defined the expression changes of both miRNAs as statistically significant (with fold change $\leq 2$ and $p<0.05$ ).

The obtained data was subsequently used to assess the diagnostic specificity and sensitivity of analyzed serum miRNAs. Diagnostic sensitivities of miR-3135a and miR-328-3p for ASD were 76.3 and $78.9 \%$, respectively. The corresponding specificities were 88.9 and $88.9 \%$, and AUC $95 \%$ confidence interval $(95 \% \mathrm{CI})$ were 0.828 (0.715-0.911\%), and $0.858(0.749-0.932 \%)$, respectively. In addition, a combined ROC analysis was done. The combined ROC curve analysis showed a better diagnostic value than individual miRNAs in ASD. Area under the ROC AUC (95\% CI) 0.858. Confidence interval (0.749-0.932). Together, these results indicate that the identified serum miRNAs, alone or in combination, can discriminate between ASD cases and TDC with high accuracy.

Identification of MicroRNAs Associated Biological Pathways. In all the 162 genes denoted as unique, validated targets in miRWalk, 71 appeared to participate in biological pathways described in the KEGG database. (Figures 5 and 6). One of the target genes we obtained, the amyloid $\beta$ precursor protein $(A P P)$, is involved in synaptic pathways. This gene encodes a membrane protein 
that undergoes proteolytic processing. In serotonergic synapses, the soluble APP $\alpha$ fragment interacts with the cyclic adenosine monophosphate (cAMP) signal transduction protein exchange factor directly activated by cAMP (EPAC) to promote neuroprotection. Another gene, solute

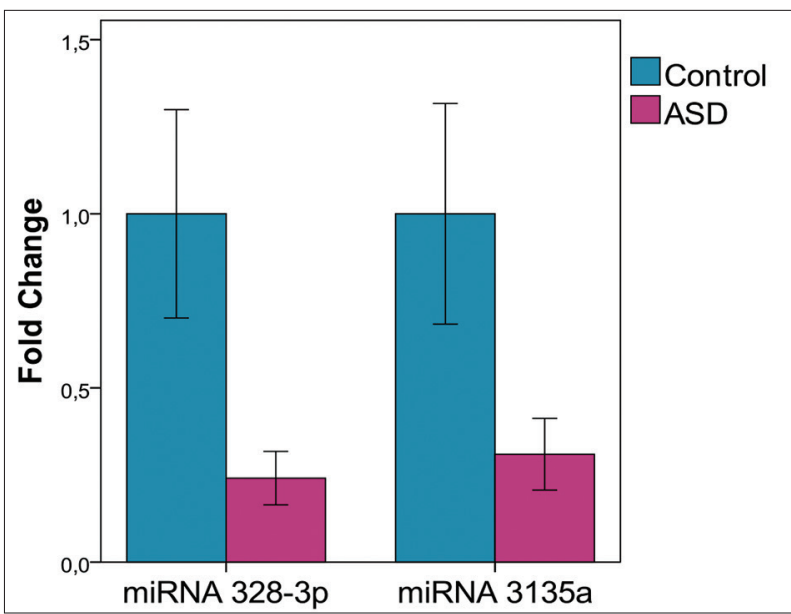

Figure 4. Fold change difference of two down-regulated serum miRNAs between the ASD and TDC groups. Data are expressed as fold change of mean $2^{-\Delta \Delta \mathrm{Ct}}$ for each miRNA after being normalized with spike-in cel-miR-39 control.

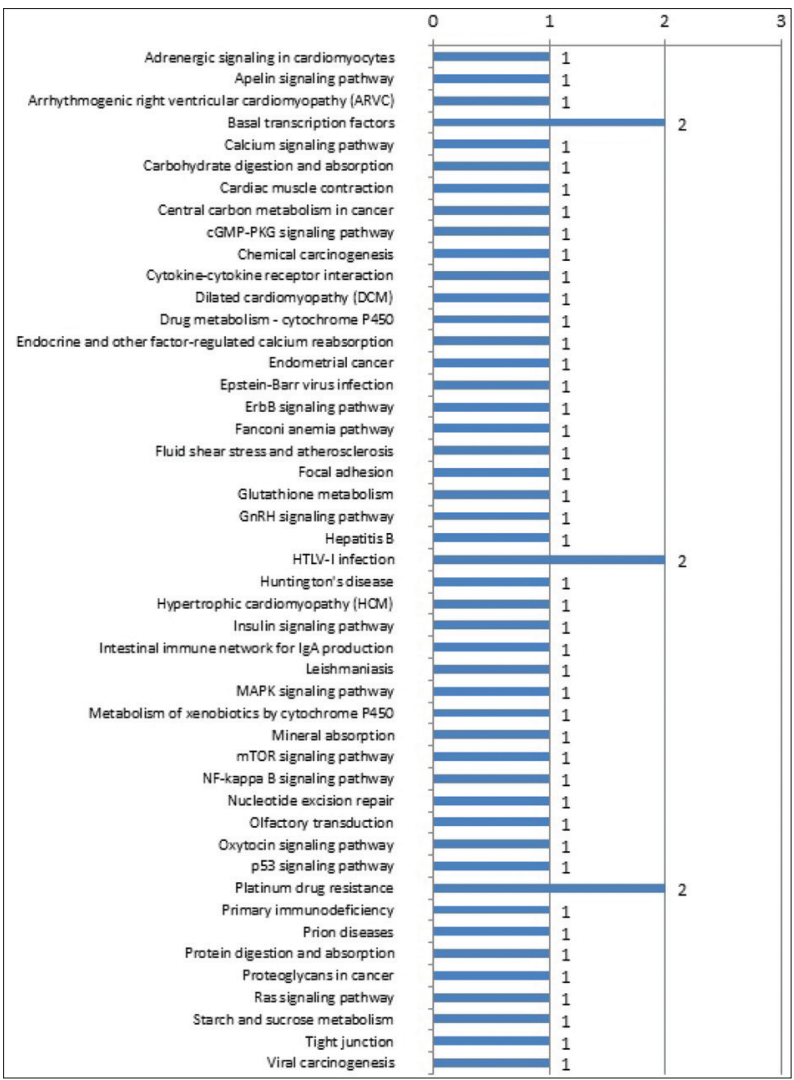

Figure 5. The KEGG pathway enrichment analysis for the targets of the identified serum miR-3135a.

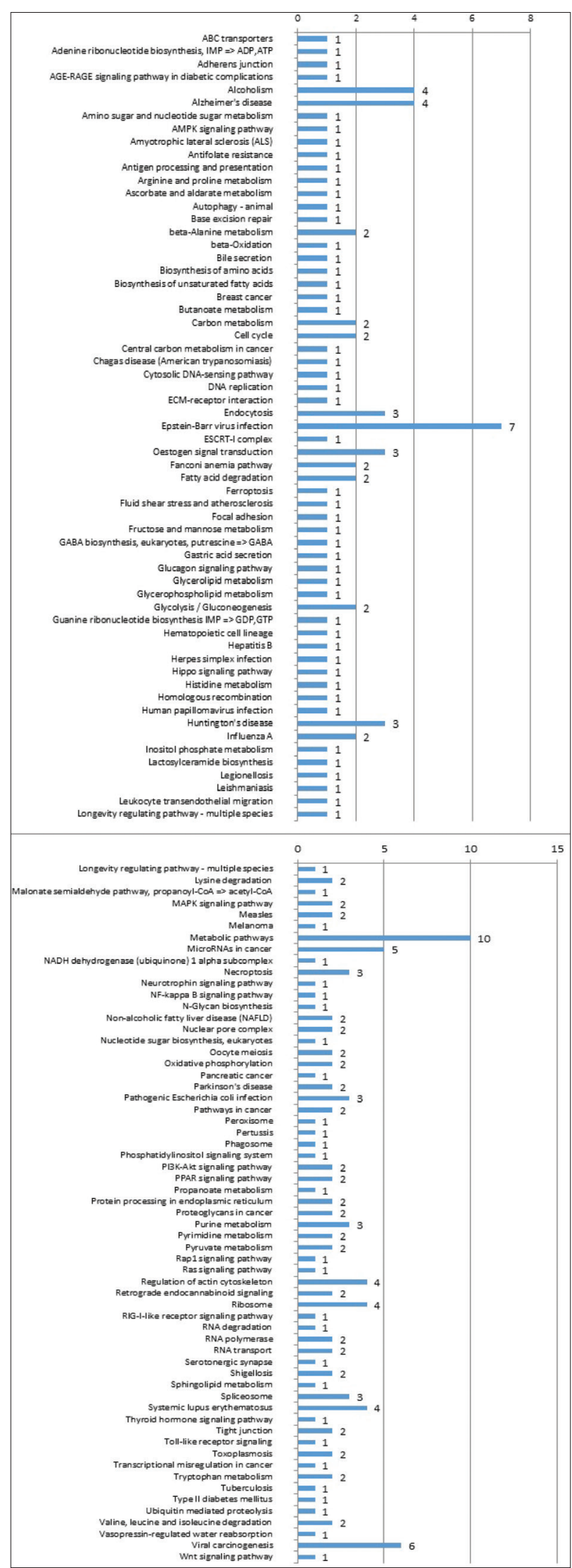

Figure 6. The KEGG pathway enrichment analysis for the targets of the identified serum miR-328-3p. 
carrier family eight member A1 (SLC8A1), participates in a specific exteroceptive transduction pathway. The SLC8 A1 protein is an $\mathrm{Na}^{+} / \mathrm{Ca}^{2+}\left(\mathrm{K}^{+}\right)$antiporter. In olfactory neurons, it has a role in membrane repolarization and annihilates the consequences of a previously occurred action potential. Another six different target genes showed involvement in neurodegenerative diseases: Huntington's, Parkinson's or Alzheimer's. Two genes: $A P P$ and $B A C E 1$ directly involved in pathogenesis of Alzheimer's disease. The amyloid precursor protein encoded by $A P P$ is cleaved by $\beta$-secretase (encoded by $B A C E 1$ ) that leaves a particular peptide responsible for amyloid plaque formation. Two proteins (DNAL1 and POLR2I) are described to take part in Huntington's disease pathogenesis in an indirect manner, while interacting with or being influenced by, the product of the Huntingtin (HTT) gene. As a result nonspecific changes in cytoskeleton organization or gene expression occur. Two other genes whose protein products take part in the mitochondrial respiratory chain NADH:ubiquinone oxidoreductase subunit A1 (NDUFA1) and NADH: ubiquinone oxidoreductase subunit V3 (NDUFV3) are described to be important not only for Alzheimer's but also for Huntington's and Parkinson's diseases. A correlation between the Alzheimer's syndrome and the reduced expression of energy metabolism genes has been well established [9]. However, the precise mechanisms in which NDUFA1 and NDUFV3 contribute to a specific neuro-development condition and their regulatory roles is yet to be clarified.

\section{DISCUSSION}

There are many reports in the literature for the potential use of miRNAs as biomarkers for neural disorders, such as Parkinson's and Alzheimer's disease [10,11]. However, only few studies have been investigating miRNA in serum samples and their importance as biomarkers is still not fully understood. Moreover, the expression profiles of miRNAs in ASD have been examined, including studies from lymphoblastoid cell cultures [12-14].

Exploring the miRNA expression patterns as potential serum-based biomarkers for ASD diagnosis is still in its infancy. The question of how exactly miRNAs regulate their target genes through fine molecular mechanisms in the context of ASD pathogenesis are not fully understood. It is currently known that individual miRNAs can have several target genes, and thus, have an impact on more than one pathway. It was found that miRNAs can regulate translation of a wide range of proteins in neurons [15] including proteins involved in neuronal migration [16], channels [17] and neuronal morphology [18]. A functioning miRNA system is obligatory in astrocytes, with loss of miRNA biogenesis that could lead to seizures and neuro-degeneration [19]. In order to examine the potential role of the two differentially expressed miRNAs, we obtained a list of their validated target genes and constructed a custom script that used the KEGG database for pathways in which the validated target genes participate. Some studies revealed that miR-3135a and miR-328-3p were involved in cancer [20,21]. Moreover, miRNA-328 dysregulation has also been associated with several complex neurological conditions, such as amyotrophic lateral sclerosis (ALS) [22], Alzheimer's [23] and prion diseases [24]. At present, there is no scientific data on the role of miR-3135a and miR-328-3p in ASD. Further research is necessary to explore how miR-3135a and miR-328-3p function in ASD.

The results of the current study present evidence that addition of circulating biomarker investigations has the potential to improve the specificity of screening and lower the age of diagnosis. Thus, we suggest that an ideal biomarker should be: 1) expressed in the brain tissues, 2) functionally or physiologically related to neurodevelopment, 3) easily measurable, and 4) differentially expressed in subjects diagnosed with ASD. In this study we identified two miRNAs in the serum in accordance with these criteria. The altered miRNAs (miR-3135a and miR-328-3p) presented in this investigation have not been identified in previous ASD research $[13,14,25]$. Moreover, functional pathway analysis of investigated serum miRNAs uncovered a significant availability of gene networks involved in neuron cellular functions or neurological disorders.

The most remarkable findings of our study were that serum miR-3135a and miR-328-3p could discriminate ASD patients from healthy controls. However, the specific pattern of these miRNAs and their appearance in the medical tests as biomarkers requires subsequent confirmation.

The discovery of new miRNAs biomarkers for ASD requires the integration of experiments from different fields including: 1) models generated from human tissues allowing for the preservation of individual genetic backgrounds so functional validation in these models may be more relevant than that in animal models, 2) induced pluripotent stem cells (iPSCs) derived neurons are promising models, but these cells have expression signatures more similar to stem cells rather than neurons [26].

Some promising candidates found so far are miR132, miR-7 and miR-195. Deregulation of miR-132 was reported in lymphoblastoid cell lines (LCLs) and postmortem cerebellar cortices from ASD patients [26-28]. The biological functions of miR-132 and its targets have been validated by many animal studies $[13,29,30]$. MiR-7 has been shown to be up-regulated in the saliva and postmortem anterior prefrontal cortices. This miRNA is located 
in the ASD-associated copy number variation $(\mathrm{CNV})$ locus [31-33] and its functional relevance has been validated using animal studies [34,35]. Deregulation of miR-195 is found in LCLs and serum samples, and it is also disrupted by an ASD-associated CNV locus $[25,13]$.

The role of miRNAs in psychiatric disorders and ASD will be further elaborated using continuously improved relevant approaches. In addition, meta-analysis of miRNAs, covering genetic variation, expression and biological function will provide valuable information for the potential role of miRNA in ASD, and this could help the diagnosis and prognosis of ASD and psychiatric disorders Moreover, miRNA biomarkers could be very useful in distinguishing of different subtypes of psychiatric disorder. Finally, our results contribute to the new course of miRNA research in ASD biology but it is only a small part of the long validation process of miRNA dysregulation in ASD patients.

\section{ACKNOWLEDGMENTS}

The authors are most grateful to the families of the participants for their collaboration. We thank Professor V. Stoyanova, Assistant Professor H. Ivanov and Professor I. Ivanov (Deparment of Pediatrics and Medical Genetics, Medical University Plovdiv, Plovdiv, Bulgaria), for their technical insights and support of this study.

Declaration of Interest. The authors report no conflicts of interest. The authors alone are responsible for the content and writing of this article.

Funding. This study was supported by H2020 Project PlantaSyst, WIDESPREAD-TEAMING, Phase 2.

\section{REFERENCES}

1. Bartel DP. MicroRNAs: Genomics, biogenesis, mechanism and function. Cell. 2004; 116(2): 281-297.

2. Mattick JS and Makunin IV. Non-coding RNA. Hum Mol Genet. 2006; 15(15): R17-R29.

3. Fombonne E. Epidemiology of pervasive developmental disorders. Pediatr Res. 2009; 65(6): 591-598.

4. Developmental Disabilities Monitoring Network Surveillance Year 2010. Principal Investigators, Centers for Disease Control and Prevention (CDC). Prevalence of autism spectrum disorder among children aged 8 years- autism and developmental disabilities monitoring network, 11 sites, United States, 2010. MMWR Surveill Summ. 2014; 63(2): 1-21.

5. Rutter M. Incidence of autism spectrum disorders: Changes over time and their meaning. Acta Paediatr. 2005; 94(1): 2-15.
6. Persico AM, Merelli S. Environmental factors and autism spectrum disorder. In: Leboyer M, Chaste P, Editors. Autism Spectrum Disorders: Phenotypes, Mechanisms and Treatments, Vol. 180. Basil, Switzerland: Karger AG. 2015: 113-134.

7. Frans EM, Sandin S, Reichenberg A, Langström N, Lichtenstein P, McGrath JJ, et al. Autism risk across generations: a population-based study of advancing grandpaternal and paternal age. JAMA Psychiat. 2013; 70(5): 516-521.

8. Ruggeri B, Sarkans U, Schumann G, Persico AM. Biomarkers in autism spectrum disorder: the old and the new. Psychopharmacology (Berl). 2014; 231(6): 1201-1216.

9. Lang W, Reimn EM, Valla J, Dunckley T, Beach TG, Grover A, et al. Alzheimer's disease is associated with reduced expression of energy metabolism genes in posterior cingulate neurons. Proc Natl Acad Sci USA. 2008; 105(11): 4441-4446.

10. Müller M, Jäkel L, Bruinsma IB, Claassen JA, Kuiperij HB, Verbeek MM. MicroRNA-29a is a candidate biomarker for Alzheimer's disease in cell-free cerebrospinal fluid. Mol Neurobiol. 2016; 53(5): 28942899.

11. Ding H, Huang Z, Chen M, Wang C, Chen X, Chen J, et al. Identification of a panel of five serum miRNAs as a biomarker for Parkinson's disease. Parkinsonism Relat Disord. 2016; 22: 68-73.

12. Ghahramani Seno MM, Hu P, Gwadry FG, Pinto D, Marshall CR, Casallo G, et al. Gene and miRNA expression profiles in autism spectrum disorders. Brain Res. 2011; 1380: 85-97.

13. Sarachana T, Zhou R, Chen G, Manji HK, Hu VW. Investigation of post-transcriptional gene regulatory networks associated with autism spectrum disorders by microRNA expression profiling of lymphoblastoid cell lines. Genome Med. 2010; 2(4): 23.

14. Talebizadeh Z, Butler MG, Theodoro MF. Feasibility and relevance of examining lymphoblastoid cell lines to study role of microRNAs in autism. Autism Res. 2008; 1(4): 240-250.

15. Kosik KS. The neuronal microRNA system. Nat Rev Neurosci. 2006; 7(12): 911-920.

16. Gaughwin P, Ciesla M, Yang H, Lim B, Brundin P. Stage-specific modulation of cortical neuronal development by Mmu-miR-134. Cereb Cortex. 2011; 21(8): 1857-1869.

17. Edbauer D, Neilson JR, Foster KA, Wang CF, Seeburg DP, Batterton MN, et al. Regulation of synaptic structure and function by FMRP-associated microR- 
NAs miR-125b and miR-132. Neuron. 2010; 65(3): 373-384.

18. Wayman GA, Davare M, Ando H, Fortin D, Varlamova $\mathrm{O}$, Cheng $\mathrm{HY}$, et al. An activity-regulated microRNA controls dendritic plasticity by downregulating p250GAP. Proc Natl Acad Sci USA. 2008; 105(26): 9093-9098.

19. Tao J, Wu H, Lin Q, Wei W, Lu XH, Cantle JP, et al. Deletion of astroglial Dicer causes non-cell-autonomous neuronal dysfunction and degeneration. J Neurosci. 2011; 31(22): 8306-8319.

20. Lu J, Xu X, Liu X, Peng Y, Zhang B, Wang L, et al. Predictive value of miR-9 as a potential biomarker for nasopharyngeal carcinoma metastasis. Br J Cancer. 2014; 110(2): 392-398.

21. Ma W, Ma C, Zhou N, Li X, Zhang Y. Up-regulation of miR-328-3p sensitizes non-small cell lung cancer to radiotherapy. Sci Rep. 2016; 6: 31651. doi: 10.1038/ srep31651.

22. De Felice B, Guida M, Guida M, Coppola C, De Mieri G, Cotrufo R. A miRNA signature in leukocytes from sporadic amyotrophic lateral sclerosis. Gene. 2012; 508(1): 35-40.

23. Provost P. Interpretation and applicability of microRNA data to the context of Alzheimer's and agerelated diseases. Aging (Albany, NY, USA). 2010; 2(3): 166-169.

24. Provost P. MicroRNAs as a molecular basis for mental retardation, Alzheimer's and prion diseases. Brain Res. 2010; 1338: 58-66.

25. Vasu M, Anitha M, Thanseem A, Suzuki I, Yamada $\mathrm{K}$, Takahashi $\mathrm{K}$, et al. Serum microRNA profiles in children with autism. Mol Autism. 2014; 5: 40.

26. Okita K, Yamanaka S. Induced pluripotent stem cells: Opportunities and challenges. Philos Trans R Soc Lond B Biol Sci. 2011; 366(1575): 2198-2207.

27. Abu-Elneel K, Liu T, Gazzaniga FS, Nishimura Y, Wall DP, Geschwind DH, et al. Heterogeneous dysregulation of microRNAs across the autism spectrum. Neurogenetics. 2008; 9(3): 153-161.
28. Talebizadeh Z, Butler MG, Theodoro MF. Feasibility and relevance of examining lymphoblastoid cell lines to study role of microRNAs in autism. Autism Res. 2008; 1(4): 240-250.

29. Ucar A, Vafaizadeh V, Jarry H, Fiedler J, Klemmt PA, Thum T, et al. miR-212 and miR-132 are required for epithelial stromal interactions necessary for mouse mammary gland development. Nat Genet. 2010; 42(12): 1101-1108.

30. Leinders M, Üçeyler N, Pritchard RA, Sommer C, Sorkin LS. Increased miR-132-3p expression is associated with chronic neuropathic pain. Exp Neurol. 2016; 283(Pt A): 276-286.

31. Shaltiel G, Hanan M, Wolf Y, Barbash S, Kovalev E, Shoham S, et al. Hippocampal microRNA-132 mediates stress-inducible cognitive deficits through its acetylcholinesterase target. Brain Struct Funct. 2013; 218(1): 59-72.

32. Hicks SD, Ignacio C, Gentile K, Middleton FA. Salivary miRNA profiles identify children with autism spectrum disorder, correlate with adaptive behavior, and implicate ASD candidate genes involved in neurodevelopment. BMC Pediatr. 2016; 16: 52.

33. Mor M, Nardone S, Sams DS, Elliott E. Hypo-methylation of miR-142 promoter and upregulation of microRNAs that target the oxytocin receptor gene in the autism prefrontal cortex. Mol Autism. 2015; 6: 46.

34. de Chevigny A, Coré N, Follert P, Gaudin M, Barbry $\mathrm{P}$, Béclin C, et al. miR-7a regulation of Pax 6 controls spatial origin of forebrain dopaminergic neurons. Nat Neurosci. 2012; 15(8): 1120-1126.

35. Pollock A, Bian S, Zhang C, Chen Z, Sun T. Growth of the developing cerebral cortexIs controlled by microRNA-7 through the p53 pathway. Cell Rep. 2014; 7(4): 1184-1196. 